\title{
VALIDATION OF A PARAMETRIC APPROACH FOR 3D FORTIFICATION MODELLING: APPLICATION TO SCALE MODELS
}

\author{
K. Jacquot, C. Chevrier, G. Halin
}

\author{
MAP-Crai (UMR 3495 CNRSMCC), ENSA Nancy, 54000 Nancy, France \\ (jacquot, chevrier, halin)@crai.archi.fr
}

Commission V, WG V/4

KEY WORDS: architectural heritage, bastioned fortifications, parametric modelling, knowledge based, scale model, 3D surveys

\begin{abstract}
:
Parametric modelling approach applied to cultural heritage virtual representation is a field of research explored for years since it can address many limitations of digitising tools. For example, essential historical sources for fortification virtual reconstructions like plans-reliefs have several shortcomings when they are scanned. To overcome those problems, knowledge based-modelling can be used: knowledge models based on the analysis of theoretical literature of a specific domain such as bastioned fortification treatises can be the cornerstone of the creation of a parametric library of fortification components. Implemented in Grasshopper, these components are manually adjusted on the data available (i.e. 3D surveys of plans-reliefs or scanned maps). Most of the fortification area is now modelled and the question of accuracy assessment is raised. A specific method is used to evaluate the accuracy of the parametric components. The results of the assessment process will allow us to validate the parametric approach. The automation of the adjustment process can finally be planned. The virtual model of fortification is part of a larger project aimed at valorising and diffusing a very unique cultural heritage item: the collection of plans-reliefs. As such, knowledge models are precious assets when automation and semantic enhancements will be considered.
\end{abstract}

\section{INTRODUCTION}

\subsection{Context}

In 2008, twelve fortified sites of Vauban were added to the Unesco World Heritage List thanks to their "outstanding universal value". In addition to these preserved strongholds, hundreds of sites were also fortified by the French marshal. A testimony of these fortified places is the collection of plansreliefs. These scale models are a perfect support to understand the dimensional realities and the urban impact of fortification throughout history. As a result, they are the primary source for any $3 \mathrm{D}$ project of fortification sites modelling.

While these scale models were made Monument Historique in the 1920s, the collection remains little known and must be better valorised. On rare occasions, exceptional exhibitions highlight some scale models but the means deployed to put in place these events are considerable. As a matter of fact, the characteristics of the collection are exceptional.

Built from the late 17th to the late 19th century, it includes a hundred models whose sizes and shapes are very different. Every plan-relief is made of pieces called tables (see Figure 1). The scale is 1:600 and their sizes may be quite large (the average surface is around $20 \mathrm{~m}^{2}$ but the biggest model is more than $150 \mathrm{~m}^{2}$ ). Some models are in a bad state due to the use of fragile materials (silk, paper, lime-wood, etc.) and the lack of protection measures. What is common to all these scale models is that they show the state of bastioned fortifications at their peak.

Although some plans-reliefs are too damaged too be exposed, the prestige of the scale model rests in part on their number. Besides, the present museum is too small to expose all of them: only 28 models are restored and presented into air-conditioned cases.

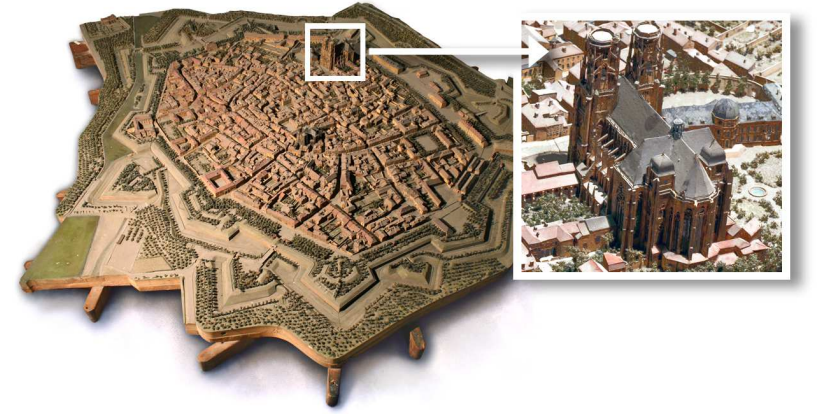

Figure 1. The main table of the plan-relief of Toul $(2.23 \mathrm{~m} \mathrm{x}$ $2.13 \mathrm{~m}$ ) and a detail showing the ten centimetres height cathedral.

Virtual scale models may resolve this dilemma. Virtual museums meet the growing expectation for enhancing, preserving and diffusing these unique pieces of cultural heritage. For example, a virtual model can be available on the net thus allowing more visibility for the collection. It can also be linked to data bases and other digital collections which is one major cultural asset. Last but not least, it is a way to promote the collection and to make people come to the museum.

\subsection{Overview of the paper}

In this the paper we present a parametric approach for the 3D modelling of fortification sites and its validation process. After a brief overview of related works both in virtual fortification and/or scale model modelling, the project background is presented (section 2). The whole approach of the project is introduced in section 3. In section 4, the fortification parametric library is described from analysis to implementation. Finally, 
the method as well as the validation is presented in section 5 and 6 before commenting the results.

\section{RELATED WORKS}

Our fortification modelling project depends in large part on plans-reliefs and data derived from the fortifications of scale models sources. Plans-reliefs are among the most complete source of data available on fortifications, but the scale is a key factor on the digitising process. On one side we have to deal with the fortification modelling and, on the other side with specific scaled data issues. Therefore we are mainly concerned with current research dealing with virtual models based on scale models given that works in fortification $3 \mathrm{D}$ reconstruction area are still limited.

\subsection{Virtual fortification and scale models}

3D modelling of Vauban's work remains largely a manual task applied to single case project as reflected by some recent projects (i.e. the "Citadelle de Marsal" by AXYZ or "Briançon" by AGP). In any event, these are projects based on full-scale fortification.

Likewise, research on historical scale models is limited. The most notable projects are the Langweil's model of Prague (Sedlacek, 2009) and the Rome Reborn (Dylla, 2009) project. The first scale model can be fragmented into 52 small parts which make handlings relatively easy. While the scale is bigger (1:480) and the geometric level of detail lower, only the digitising was automated. About 200 people were involved in the making of the virtual model of the scale model of Prague. The Rome Reborn project is based on a 1:250 scale model. A 3D survey gives a basic geometrical layout of all the elements of the Ancient Rome. Apart from the manually modelled famous buildings, most of the modelling was done using a procedural engine which allows the creation of wide urban environments satisfying architectural cannons of Rome at the time. Even if these last works are not intended to depict large fortification sites, they demonstrate the need of specific approaches when it comes to use small-scale data.

\subsection{Cultural Heritage reverse engineering}

Reverse engineering approach in Cultural Heritage begins with the digitising of an existing object. The scanned data are then used as a support to model the different parametric components which are part of the scanned object. The use of parametric components allows the modelling process to be faster. The size of created objects or environment is no longer based on the time spent in modelling. Finally, it also makes the semantic enhancement easier such as in BIM practise.

By using specific domain knowledge (i.e architectural knowledge), it is possible to improve the reconstruction process. Theoretical literature like Renaissance treatises seeks to give an exhaustive account of every aspects of architecture. Hence, missing or hidden parts from 3D surveys are often predictable since they are well documented. Therefore, Murphy's HBIM (2011) includes modelling onto point cloud surveys thanks to a parametric library based on reference texts. However, the parametric objects adjustment remains a manual task. Other values of HBIM/BIM approach are interoperability and semantic data support. This approach is also ideal for 2D/3D documentation: technical drawings, orthographic projection, sections, details and 3D models with various level of detail according to visualisation requirements (Boeykens, 2011).
Knowledge based reconstruction projects use thesauruses or semantic nets (Cantzler and al., 2002). Knowledge models defines both the attributes of the features from a specific domain and the hierarchical and semantic relations between them. Once the data from 3D surveys are semantically labelled or segmented (i.e. slope, hip, eave, valley, ridge, etc. for the study of roofs), the automatic reconstruction is possible (Chevrier, 2010). Finally, thesauruses can be used to integrate semantic contents in the resulting geometrical model $(\mathrm{Pu}$ and Vosselman, 2009).

\subsection{Project Background}

Some research has been under way for the modelling of the town and campaign parts of the plans-reliefs. Besides typical problems in data acquisition process (self-occlusion or rounded edges), our early works showed that the digitising of scale models has specific issues. The size, scale and accuracy of the models are indeed out of range of 3D scanners (Remondino, 2011). For instance, the woodworks on the whole model are around a quarter of a millimetre. Not only do we have to deal with small details, we also have to work on very large scale model. Moreover, the age ( 350 for the oldest) and the quantity of plans-reliefs bring specific difficulties. In summary, wear and tear, high level of details on large surfaces and automation are the three most influential factors on plans-reliefs digitising.

\section{APPROACH}

Figure 2 illustrates the different steps of the whole approach for the $3 \mathrm{D}$ reconstruction of the bastioned fortification. First, we have to study the fortification science in its theoretical aspects to define as thoroughly as possible all the fortification elements. This has been made easier by the existence of thesauruses (Chatenet and al., 2000) and bastioned fortification treatises (Figure 2, a). Over its 250-year history, fortification art has rapidly evolved to meet progress of artillery. This led to a wide corpus of theoretical literature that had to be analysed and refined (Jacquot, 2011). Once formulated into knowledge models, particularly geometrical knowledge model, we have identified and defined every fortification components and their relationships (Figure 2, b). A library of parametric components is then implemented into a prototype (c). We use Grasshopper, a visual dataflow programming language (VDPL) editor, to implement and manually adjust the different components on a relevant corpus of fortifications sites (d). Easy to use, VDPL do not require special programming skills whereas they can achieve the creation of complex 3D parametric scenes in short time span. Thus, users specify a sequence of relationships and operations - in the form of links and nodes - to automate the construction of geometry. As for the fortification sites, we use several digitised sources (e), from 3D surveys of plans-reliefs (acquired by photogrammetry and lasergrammetry) to scanned maps from the $17^{\text {th }}$ and $18^{\text {th }}$ centuries, but also scale models construction plans (f). Finally, a validation method is defined (g). Once validated, the parametric library will be used in a process aiming at the automation of the adjustments.

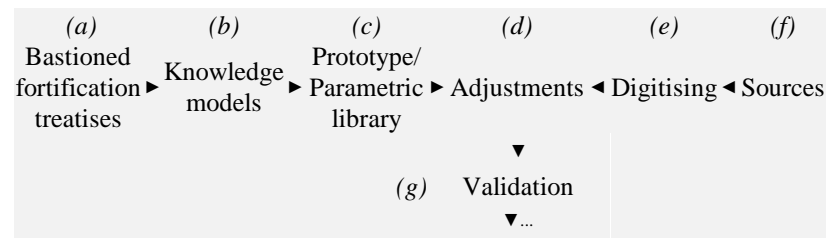

Figure 2. Main steps of our approach. 


\section{THE PARAMETRIC LIBRARY}

\subsection{Study of bastioned fortification treatises}

Empirical analysis of bastioned fortification reveals the importance of geometry for the construction of bastioned works. The polygonal patterns of fortifications are designed to prevent dead angles which could benefit the enemies. Size and placement of every fortification work is relative to armament ranges as "fortifications consist of lines and angles, which have various names according to their various offices" (Hutton, 1815)

Like architecture, fortification has been theorised in treatises in order to gather and transmit specific knowledge. Hundred of treatises were published until mid- $19^{\text {th }}$ century but critical reviews of this literature are still rare. However, recurrent elements are present in the selected subset of treatises: geometry, fortification principles and history, construction methods for all works by top engineers, etc.

Classification schemes like thesaurus allow us to use all along the project a controlled and structured vocabulary in which concepts are clearly defined and their relationships are made explicit (Jacquot, 2012). For now, we use knowledge management of bastioned fortification domain to understand and synthesise the geometric nature of fortifications.

\subsection{Grasshopper implementation}

The knowledge models resulting from the analysis of the bastioned fortification treatises highlight the importance of regulating lines. Such lines are "used to control proportion and placement of elements" (Ching, 2012) and other lines from the fortification system can relate to them. Most of the fortifications works are designed with these lines which are described by specific methods. For example, the basic fortification entities such as the bastioned front of the city wall, the demi-lunes and the counterscarp have their own methods to draw their main line.

As described in Figure 3, these works (approximately 80\%) can be modelled by using trajectories along which 2-dimensional profiles are swept orthogonally. Moreover, it is a hierarchical process: once the city wall is modelled, all the other trajectories and works are created based on the trajectory used for the city wall. A library of components consisting of regulating lines, profiles and specific sweep operations is only required to model a significant part of bastioned fortification.

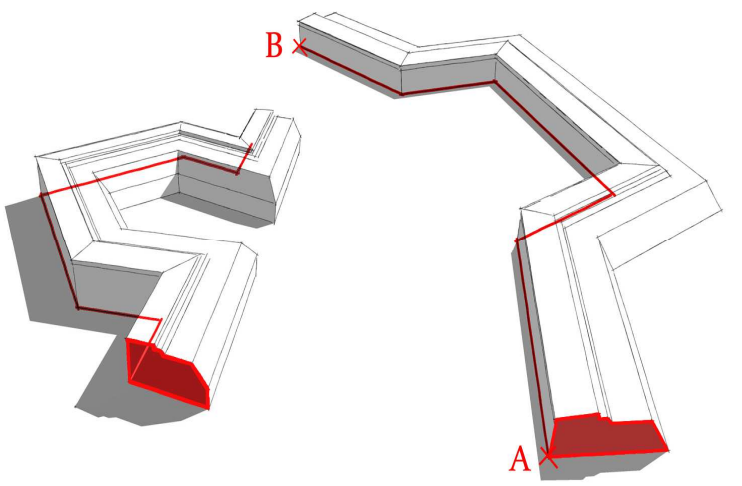

Figure 3. Profiles and trajectories lines for a bastion (left) and a bastioned front (right).
For now, these components are created and manually adjusted in Grasshopper to the different sources available. The aim is to test, complete and to improve the components and their parameters.

\subsection{Principle of use}

Both ends of a city wall front line (A and B in Figure 3) must be manually placed to allow the first operation to begin. Figure 4 depicts the components used on Grasshopper to achieve the modelling of a bastioned front (Figure 3, right). The component 1 is the city wall front line operation which is linked to our sweep component (3). To create the 3D front, it is also necessary to specify a profile (2) which can change from one work to the other.

The whole fortification system can be modelled in the same way. The previous geometry is used as a parameter for new regulating line operations in order to identify automatically their location. This trajectory is in turn used as a parameter of a sweep component alongside a profile to create a new 3D fortification work.

Only some values of parameters have to be modified to correct regulating line operations. Thus, manual adjustments are made based on default parameters. Additional values ranges are defined to allow specific adjustments for each case. Special attention is paid to keep the narrowest range of values possible.

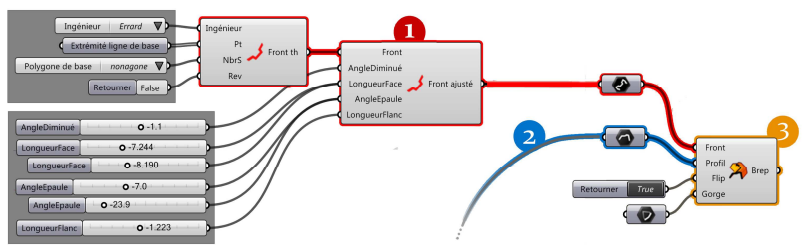

Figure 4. Grasshopper clusters for the modelling of a part of the city wall. In the far left, several parameters (angle and length values) of the city wall front line (1) have been adjusted before the resulting line is linked in conjunction with a profile (2) as a parameter of the sweeping component (3).

The different operations specified in Grasshopper are organised to match specific fortification entities such as city wall front line, front profile or sweep tool, as defined in the knowledge models.

\section{METHOD FOR THE VALIDATION OF THE PARAMETRIC LIBRARY}

To validate the parametric library, a specific process has also been defined and implemented. We choose to use and adapt Goodchild's technique (1997) because the evaluation of our resulting data is close to geographical accuracy issues like linear features matching. Line abstractions are widely used to represent linear aspect of many geographical entities like road, rivers, coastlines, etc. and so research in geographical information science is abundant. Among the problems facing them, measuring tools and features definitions are the ones that we have to deal with. Popular evaluation methods are based on points-correspondence or/and on buffers like Goodchild's.

By comparing our 2-dimensional trajectories to reference lines, we can assess the accuracy of our graphical representation and thereby validate our method. Each trajectory must be part of a buffer zone of specific width around the reference lines as illustrated in figure 5 . 


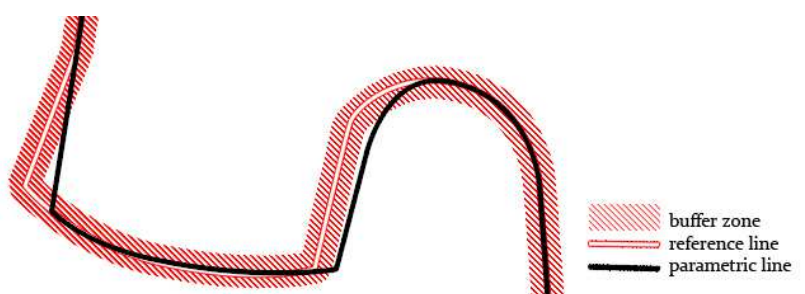

Figure 5. To validate the parametric line, we assess the percentage of this line inside the buffer zone.

To determine the width, we use an exacting standard of the IGN, the national institute of the geographic information in France that defines the dimensional precision of maps. Taking into account technical errors (such as parametric components) and human errors (such as manual picking of points), the tolerance is fixed at $0.1 \mathrm{~mm}$ measured on the map regardless of its scale (i.e. $6 \mathrm{~cm}$ on the ground for a map at 1:600). It should be noted that this value is among the more stringent (the allowable error distance calculated by the USA for a 1:1000 map is around 0.85 metres).

In our case, it means that our linear features must be part of a buffer zone of maximum of $0.1 \mathrm{~mm}$ around the reference lines. Thus, we can calculate the percentage of parametric lines within the buffer zone thanks to Grasshopper component which solve intersection events for a surface (the buffer zone) and a curve (the parametric line). The ratio is determined by deducing the total length of the intersected parametric curves from the total length of the initial curve.

\section{EXPERIMENTS}

\subsection{Corpus of fortification sites}

The test group is composed of six strongholds from the French Northeast Border which present different degrees of regularity as demonstrated in the following figure.

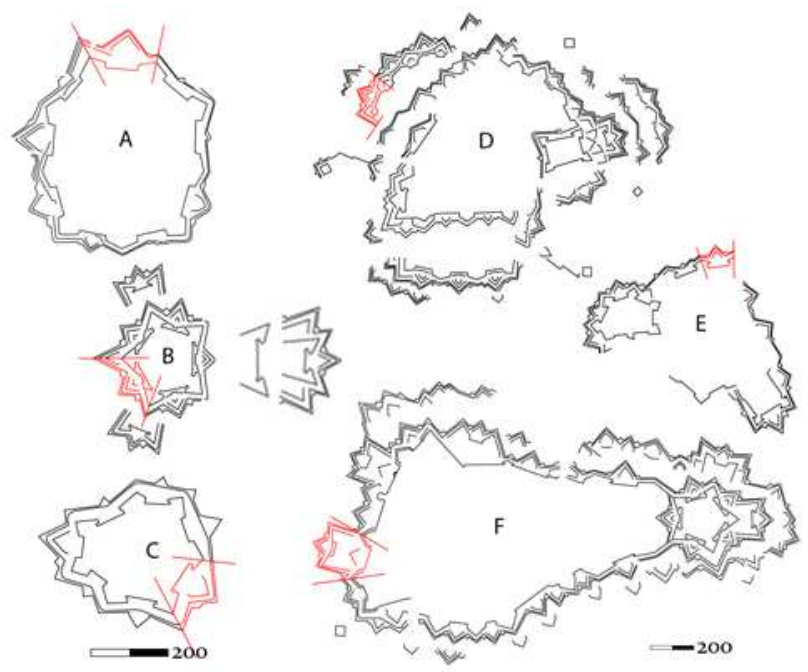

Figure 6. Regulating lines of the fortified sites of Toul (A), Huningue (B), Marsal (C), Metz (D), Verdun (E) and Strasbourg (F). The scale bars show the distance of 200 toises (approximately 190 metres). The red areas represent a front.

For the city of Toul (A) Marsal (C) and specially Huningue (B), the fortifications are almost a regular polygon given the facts that reliefs, rivers or previous fortifications have little impact on theoretical regulating lines. In contrast, for Metz (D), Verdun (E) and, above all, Strasbourg (F) the surroundings are complex which lead to sprawling fortifications made of forts and outworks. Thus, we have a contrasting sample group of fortification systems for testing our approach.

As regards the experimented components, we mainly focus on the eight most common works, namely the city wall and its bastioned fronts (33\% of the works), the demi-lune (23\%), the counterscarp $(30 \%)$, the tenaille $(8 \%)$, the counterguard $(2 \%)$ (Figure 7) and some outworks.

Components are created thanks to theoretical parameters values which are used in specific order like a sequence of instructions. Parametric adjustments have to be operated in the same order as this sequence; otherwise components adjustments can be tricky.

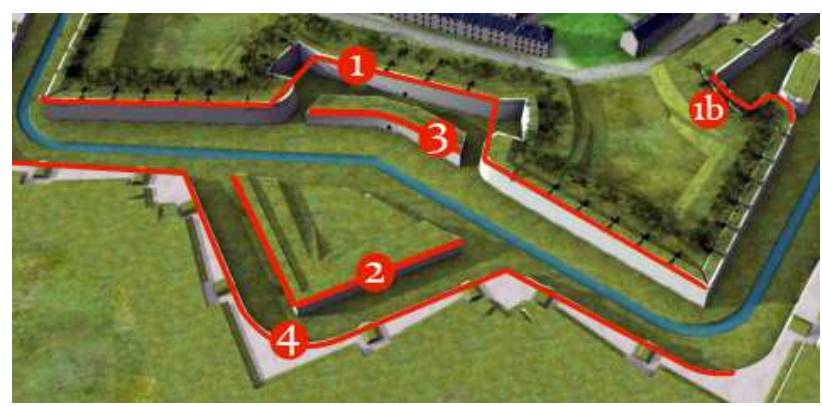

Figure $7^{*}$. One bastioned front of the city wall (1), an orillon (1b), a demi-lune (2), a tenaille (3), a counterscarp (4) and their main lines in red.

\subsection{Results}

The results for the first regulating line (i.e. the city wall front) in most cases are very close to the reference linear features (Table 1). The average percentage for the 71 parametric bastioned fronts (column 1) of the selected corpus of plans-reliefs is around $95.5 \%$ even for more complex walls where orillons (erashaped projection at the flank of bastions: 1b in Figure 7) are present like on the citadel of Verdun or Huningue. Both the parametric counterguards, demi-lunes and the tenailles (column 2,3 and 4) show good results $(\sim 90 \%)$. On Toul case, the tenailles are far more irregular to be modelled with a generic component. Counterscarps (column 4) can vary greatly as their shapes depend both of the bastioned front and the counterguard/demi-lune operations.

Given the fact that components are relative to others, the adjustment of component tends to be more difficult because it is based on previous parametric operations. The further the works are from the city, the more difficult their parametric modelling become. This is the main reason why the average percentages are decreasing.

Furthermore, we noticed that even on highly irregular fortification systems (Metz, Verdun, and Strasbourg), the parametric library is well adapted. Fortification works are highly generic shapes: each bastioned front (see 1 on figure 7) is a support for one or several other works. When considered undependably, these fronts and theirs works remain largely regular. It is the combination of fronts that may give the fortification system its apparent irregularity as depicted in figure 6. These geometrical patterns are more or less complex according to their environment.

\footnotetext{
* Adapted from an illustration by Art Graphique et Patrimoine.
} 


\begin{tabular}{|c|c|c|c|c|c|}
\hline Toul & 99.9 & 100 & 56.5 & N/A & 75 \\
\hline Marsal & 98.9 & N/A & 98.8 & N/A & 58 \\
\hline Huningue & 86.8 & 100 & 93.4 & 89.2 & 86.5 \\
\hline Metz & 97.4 & N/A & 96.7 & 82.3 & 97.2 \\
\hline Verdun & 97.7 & N/A & 91.7 & N/A & 93.6 \\
\hline Strasbourg & 92 & 99.7 & 91.5 & 84.6 & 81.2 \\
\hline Average & 95.5 & 99.9 & 89.1 & 85.4 & 81.9 \\
\hline
\end{tabular}

Table 1. Accuracy of each parametric component once adjusted on the sources in percentage of parametric line within the buffer zone.

\subsection{First corrective measures}

The shortest value range and the ideal default value are also studied in order for the parametric library to be no longer theoretical but practical. This review is useful for new manually adjustments as it aims to reduce user intervention but also for the subsequent automatic adjustments as component constraints become more restrictive. To this end, we calculate the quartiles $\mathrm{Q}_{1}, \mathrm{Q}_{2}$ and $\mathrm{Q}_{3}$ for each parameter. These three points divide the data set into four equal groups. As the first parameter of the city wall front is already used 136 times in the six fortified sites reconstruction, we can speed up its adjustment process by using the median $\left(\mathrm{Q}_{2}\right)$ of the data set as a default value for future use. The interquartile range, (i.e. the difference between the upper and lower quartiles $\mathrm{Q}_{3}$ and $\mathrm{Q}_{2}$ ) can be used to prioritise a restricted value range for each parameter.

\section{CONCLUSION}

Virtual models of architecture heritage such as fortification make inevitable the use of a wide variety of sources. Among these data, the plans-reliefs are the most precious assets because they depict fortifications at the peak of their developments.

Starting from traditional 3D surveys, the digitised campaigns and their results reflect how complex and unique are the plansreliefs and the ambitious fortification systems. The 3D modelling of fortification (Figure 8) can only be done thanks to the use of specific knowledge of the domain. Bastioned fortification treatises give us access to the knowledge required to implement a parametric library prototype of fortification components. Conducted primarily on data derived from the fortifications of scale models, the parametric components are now manually adjusted successfully. The assessment process intended to validate the parametric approach is based on a proven GIS method. The percentage levels in the adjustment results are already high.

Several areas for further development are now explored like the automation of the reconstruction process starting with a fully automated adjustment step. For now, some tests have been done with Galapagos optimisation algorithms - an evolutionary solver included in Grasshopper - to improve the reconstruction. For components with complex parameters, it allows us to automate the adjustment with the added benefit of high percentages of accuracy. Nevertheless, VDPL editors suffer from known limitation such as iterations (Janssen and al., 2011) which also raises the question of the continuation of the project with Grasshopper.
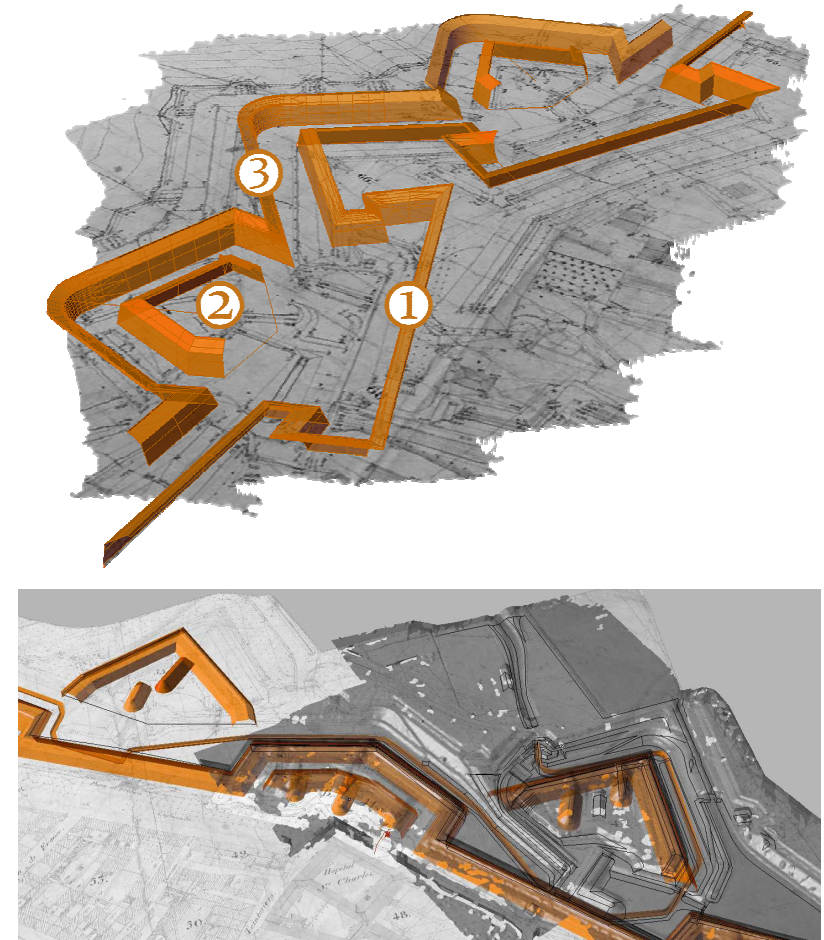

Figure 8. 3D parametric model of some fortification works placed on digitised maps and plans-reliefs: a bastioned front (1), a demi-lune (2) and a couterscarp (3).

Finally, in order to be fully automatic, the digitized sources such as 3D point clouds from photogrammetry or lasergrammetry have to be also automatically segmented. The defined knowledge models can be used for this purpose with a long-term perspective.

\section{REFERENCES}

AXYZ Images, 2010. Video "Citadelle de Marsal", Bordeaux, France http://www.dailymotion.com/video/xc4min_citadellemarsal_creation (31 Jan. 2013)

Art Graphique et Patrimoine, Service du Patrimoine de la ville de Briançon, 2006. "Briançon, ville forte du Dauphiné", Joinville-le-Pont, France.

Boeykens S., 2011. Using 3D Design software, BIM and game engines for architectural historical reconstruction. Designing Together, pp. 493-509.

Cantzler H., Fisher R., Devy M., 2002. Quality enhancement of reconstructed 3D models using coplanarity and constraints, Pattern Recognition, pp. 34-41.

Chatenet M., Verdier H., 2000. Thesaurus de l'architecture, Paris, Patrimoine : Sous-direction des études, de la documentation et de l'inventaire, Direction de l'architecture et du patrimoine, Ministère de la culture et de la communication, (Documents \& méthodes, no 7).

Chevrier C., Jacquot K., Perrin J.-P., 2010. 3D Modelling of a Town Scale Model. Proceedings of EuroMed 2010, 3rd International Conference dedicated on Digital Heritage, Limassol, Cyprus, pp. 99-107. 
Ching F.D.K., 2012. Architecture: Form, Space, and Order. John Wiley \& Sons, pp. 306-307.

Dylla K., Frischer B., Müller P., Ulmer A. and Haegler S., 2009. Rome Reborn 2.0: A Case Study of Virtual City Reconstruction Using Procedural Modeling Techniques. Proceedings of the CAA Conference. pp.62-66.

Goodchild M.F., Hunter G.J., 1997. A simple positional accuracy measure for linear features, International Journal of Geographical Information Science. pp. 299-306.

Hutton C., 1815. A Philosophical and Mathematical Dictionary, London

Jacquot K., Chevrier C., Halin G., 2011. Study of the Fortification of old scale models in order to automate their 3D modelling. eCAADe Conference Proceeding. pp. 915-924.

Jacquot K., Chevrier C., Halin G., 2012. Une Ontologie pour la Modélisation des Fortifications Bastionnées, SCAN Proceedings. pp. 201-210.

Janssen P., Chen K.W., 2011. Visual Dataflow Modelling: A Comparison of Three Systems. Proceedings of the 14th international conference on Computer Aided Architectural Design, Liège, Belgium. pp.802-816.

Murphy M., Mcgovern E., Pavia S., 2011. Historic Building Information Modelling - Adding Intelligence to Laser and Image Based Surveys. Proceedings of the 4th ISPRS International Workshop.

Remondino F., 2011. Heritage Recording and 3D Modeling with Photogrammetry and 3D Scanning, Remote Sensing. pp. 1104-1138.

Sedlacek D., Zara J., 2011. The Langweil Model of Prague - a Challenge for State-of-the-art 3D Reconstruction Techniques. Poster on Eurographics 2011. pp. 5-6. 